(Bildung von $q$-Gamon $\mathrm{A}=\mathrm{A}+$, Bildung von $\hat{\jmath}-\mathrm{Ga}-$ mon $\mathbf{A}=\mathbf{A} \hat{\delta}$, Reaktionsfähigkeit der $+q$ auf $\mathbf{A} \hat{\delta}$, Reaktionsfähigkeit der $\hat{\delta} \hat{o}$ auf Aㅇ). Zwei dieser Faktoren, nämlich die Abgabe von A $\hat{\delta}$ und die Reaktionsfähigkeit der $\hat{\delta} \hat{\delta}$ auf $\mathrm{A}+\mathrm{q}$ sind lichtabhängig und verdecken daher eine etwa vorhandene lichtabhängige Gamonwirkung B. Unsere Versuche über den Einfluß des Lichtes auf die Kopulation ${ }^{2}$ machen die Annahme eines weiteren lichtabhängigen Kopulationsfaktors (Gamon B) überflüssig. Sie sind durch die Lichtabhängigkeit der Gamonwirkung A vollständig zu erklären. Die $q$ $\uparrow$ brauchen kein Licht, um kopulationsfähig $\mathrm{zu}$ werden, für die Kopulationsfähigkeit der $\widehat{\jmath} \hat{o}$ ist dagegen das Licht ein wichtiger Faktor. Diese Lichtabhängigkeit ist zwar nicht obligatorisch, da Kopulationen prinzipiell auch im Dunkeln möglich sind. Wenn man die zusammengegebenen Zellen von beiden Geschlechtern längere Zeit stehen läßt, treten meist auch im Dunkeln Gruppen und Pärchen auf. Welche Faktoren hierbei an Stelle der Lichtwirkung treten, ist noch ungeklärt. Der Kopulationsverlauf ist aber im Dunkeln mindestens um mehrere Stdn. verzögert.

Besonders aufschlußreich sind die Licht-Dunkel-

2 H. F örster u. L. Wi es e, Z. Naturforschg. 9 b, 470 [1954].
Versuche an $\hat{\jmath} \hat{\jmath}$. Im Licht voll reaktionsfähig gewordene $\hat{\jmath} \hat{\jmath}$ verlieren beim Übertragen ins Dunkle ihre Reaktionsfähigkeit auf das A -Gamon. Übereinstimmend damit ist auch die Pärchenbildung von Kulturen, die im Licht voll kopulationsfähig geworden sind und dann im Dunkeln zusammengegeben werden, stark verzögert gegenüber den Kontrollen. Die Gamone Aô und $A \hat{q}$ sind bereits im Licht abgegeben, und sie bleiben nach Dunkelstellen wirksam vorhanden. Diese Versuche stehen im Widerspruch zu den Filtratversuchen von Moewus. Nach seinen Versuchen sollen Filtrate kopulationsfähiger Zellen das Licht ersetzen, indem sie die Zellen im Dunkeln kopulationsfähig machen. Das Gamon B müßte danach im Dunkeln wirksam bleiben. In unseren Versuchen nimmt aber die Kopulationsfähigkeit nach Dunkelstellen sehr rasch ab. Dieser Effekt läßt sich ohne weiteres auf das Verhalten der $\hat{\jmath} \hat{\jmath}$ gegenüber Gamon A우 zurückführen. Testet man allerdings solche $\hat{\jmath} \hat{b}$, die im Licht reaktionsfähig geworden sind und dann dunkel gestellt wurden, gegen reaktionsfähige Hell- +9 , so tritt sofort Gruppenbildung ein.

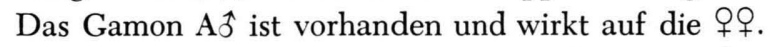
Bei der Beobachtung unter dem Binokular werden die $\widehat{\delta}$-Zellen belichtet und infolgedessen nach kurzer Zeit wieder voll kopulationsfähig.

\title{
Der kontraktile Myosinfaden aus glatter Muskulatur
}

\author{
Von Dieter Dörr und Hildegard Portzehl \\ Aus dem Physiologischen Institut der Universität Tübingen \\ (Z. Naturforschg. 9 b, 550-555 [1954]; eingegangen am 15. Juli 1954)
}

1. Aus dem glatten Fußmuskel von Anodonta lassen sich in ähnlicher Weise geordnete Fäden aus mehrfach gereinigtem Aktomyosin herstellen wie aus den quergestreiften Muskeln des Kaninchens.

2. Diese Fäden haben mit den Aktomyosinfäden aus Kaninchenmuskeln das niedrige Spannungsmaximum, die niedrige Zerreißfestigkeit und die sehr geringe Verkürzungsgeschwindigkeit gemeinsam. In diesen drei Eigenschaften unterscheiden sich beide Arten der Fadenmodelle von den Fasermodellen, die aus den gleichen Muskeln hergestellt sind.

3. Ferner zeigen die Fadenmodelle aus Anodonta das gleiche Verkürzungsmaximum, die gleiche Empfindlichkeit gegen Salyrgan und die gleiche Abhängigkeit der Kontraktion von der ATP-Spaltung und der Erschlaffung von der Hemmung der ATP-Spaltung wie alle Arten von Faser- und Fadenmodellen.

4. Die Fadenmodelle aus Anodonta-Aktomyosin unterscheiden sich von den Fadenmodellen aus Kaninchen-Aktomyosin in der Temperaturabhängigkeit der Spannung in der Unvollständigkeit der Einstellung des Spannungsgleichgewichts, wenn die gleiche Temperatur einmal von oben und einmal von unten erreicht wird, in der Lage des ATP-Optimums der Kontraktion und ihrer Beeinflußbarkeit durch Pyrophosphat.

5. Da beide Fadenmodelle sich in den letztgenannten Eigenschaften ebenso verhalten wie die Fasermodelle aus den gleichen Muskeln, müssen diese Eigenschaften auf Besonderheiten des jeweiligen Aktomyosins beruhen und nicht auf Besonderheiten der übergeordneten Struktur der Muskeln, die nur in den Fasermodellen noch erhalten ist.

* Mit Unterstützung des Unitarian Service Committee und des Oberlaendertrust, Philadelphia. 
I.

Was enn Skelettmuskeln, glatte Muskeln und Zellen aus Gewebekulturen mit wässerigen Glycerinlösungen extrahiert werden, so erlischt die Erregungsfähigkeit aller dieser Gebilde, weil ihre Membranen zerstört sind. Und ebenso erlischt ihre Restitutionsfähigkeit, weil die Betriebsstoffe und Fermente des Stoffwechsels vollständig oder weitgehend extrahiert sind. Die kontraktilen Strukturen der Zellen und Muskeln bleiben dagegen erhalten und können durch Adenosintriphosphat (ATP) in Betrieb gesetzt werden 1, 2, 3. Die ATP-Kontraktion solcher Strukturen stimmt in vielen Eigenschaften quantitativ überein mit der vitalen Kontraktion der Zellen 2 oder Muskeln 4, 5, aus denen diese Faser- oder Zellmodelle hergestellt sind. Infolgedessen sind solche Eigenschaften (z. B. die maximale Spannung und ihre Temperaturabhängigkeit) für Modelle verschiedener Herkunft ebenso verschieden wie für das lebende Ausgangsmaterial. Andere Eigenschaften, wie z. B. das Verkürzungsmaximum, sind für alle Modelle gleich - unabhängig davon, ob das Verkürzungsmaximum des lebenden Ausgangsmaterials ganz verschieden war ${ }^{6}$.

Es ist sehr wahrscheinlich, daß die Verschiedenheiten der Vitalkontraktion, die beim Übergang zum extrahierten Modell verschwinden, nicht auf den fundamentalen Eigenschaften der kontraktilen Proteine beruhen, sondern auf der Art, wie diese Proteine in die komplizierte lebende Gesamtstruktur der einzelnen Muskelarten (oder Zellarten) eingebaut sind. Aber auch die Verschiedenheiten der Vitalkontraktion, die beim Übergang zum mit WasserGlycerin extrahierten Modell nicht verschwinden, brauchen nicht auf Fundamental-Eigenschaften der jeweiligen kontraktilen Eiweißkörper zu beruhen. Denn der größte Teil der Strukturverschiedenheiten des lebenden Ausgangsmaterials bleibt bei der Wasser-Glycerin-Extraktion erhalten. Wenn dagegen brauchbare kontraktile Modelle aus zuvor gelöstem und gereinigtem kontraktilen Protein hergestellt werden, so bleiben nur noch die Strukturunterschiede übrig, die auf den Verschiedenheiten des Aktomyosins verschiedener Provenienz selbst beruhen. Die kontraktilen Eigenschaften von wohlgeordneten Aktomyosinfäden verschiedener Herkunft konnten aber bisher nicht verglichen werden, weil geordnete Fäden bisher nur aus dem gereinig-

1 H. H. W e b e r, Biochim. biophysica Acta [Amsterdam] 12, 150 [1953].

$2 \mathrm{H}$. H of $\mathrm{fm}$ a $\mathrm{n}$ - B e r li n g, Biochim. biophysica Acta [Amsterdam] 14, 182 [1954].

$3 \mathrm{H}$. P o rtzeh l, Biochim. biophys. Acta [Amsterdam] 14, 195 [1954].

4 H. H. W e ber u. H. Portzehl, Erg. Physiol. biol. Chem. exp. Pharmakol. 47, 369 [1952]. ten Aktomyosin von Kaninchenmuskulatur hergestellt wurden ${ }^{7,8}$. Sie können aber auch nach dem gleichen Verfahren aus der glatten Muskulatur des Fußes von Anodonta hergestellt werden. Es werden die kontraktilen Eigenschaften dieser Fadenmodelle aus glatter Muskulatur beschrieben und mit den kontraktilen Eigenschaften des Aktomyosinfadens aus Skelettmuskel einerseits und den entsprechenden Eigenschaften der Fasermodelle aus glatten Muskeln von Anodonta andererseits verglichen.

II.

Die maximale Spannung solcher Fadenmodelle von Anodonta-Muskel ist der Spannung der Fadenmodelle aus Kaninchenmuskulatur annähernd gleich:

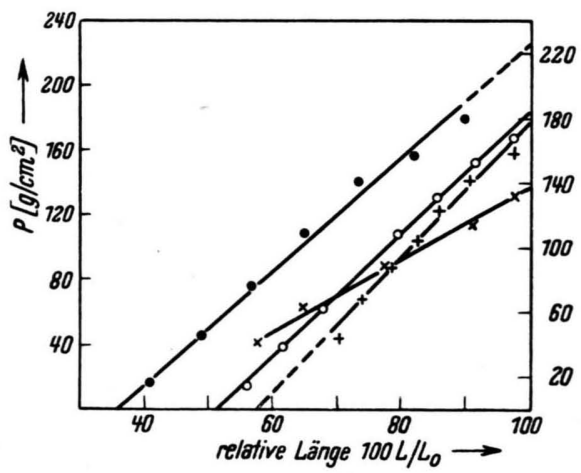

Abb. 1. Abhängigkeit der maximalen Spannung der ATPKontraktion von der Fadenlänge. Die Versuchspunkte der einzelnen Kurven bedeuten Einzelkontraktionen desselben Fadens (4 Fäden). 1 bis $2 \cdot 10^{-3}-m$. ATP, $8 \cdot 10^{-4}-m$. $\mathrm{MgCl}_{2}, 0,04-m . \mathrm{KCl}, 0,01-m$. Phosphat, $p_{\mathrm{H}} \sim 6,9$. T. $20^{\circ} \mathrm{C}$.

$\sim 200 \mathrm{~g} / \mathrm{m}^{2}$ (Abb. 1). Die Anodonta-Fadenmodelle gleichen den Kaninchen-Fadenmodellen auch in ihrer Neigung, unter ihrer eigenen Spannung bei Standardlänge zu zerreißen. Auch die Maximalspannung der Anodontafäden nimmt mit fortschreitender Verkürzung etwa geradlinig ab.

Die angegebenen und in Abb. 1 gezeigten Spannungen sind Minimalwerte, weil auch beim Fadenmodell aus Anodonta offensichtlich die W a r b u r g sche Grenzschichtdicke für ATP überschritten ist:

5 H. H. Weber u. H. Portzehl, Progress Biophysics 4, 60 [1954].

6 H. H. W e b e r, Harvey Lecture, Oktober 1953, im Druck.

7 H. Portzehl u. H. H. W eb er, Z. Naturforschg. 5b, 123 [1950]; H. P ortzehl, Z. Naturforschg. 6 b, 355 [1951].

8 T. Hayashi, Federation Proceedings 10, 61 [1951]. 
denn Abb. 2 zeigt, daß die maximale Spannung bei 95\% der Standardlänge mit abnehmendem Fadendurchmesser bis herunter zu einem Durchmesser von $55 \mu$ zunimmt. Das ist ebenso wie bei dem Fadenmodell aus Kaninchenmuskel, obwohl die Spaltungsrate des Aktomyosins aus Anodonta viel kleiner ist ${ }^{9}$. Denn auf der anderen Seite ist auch die angewen-

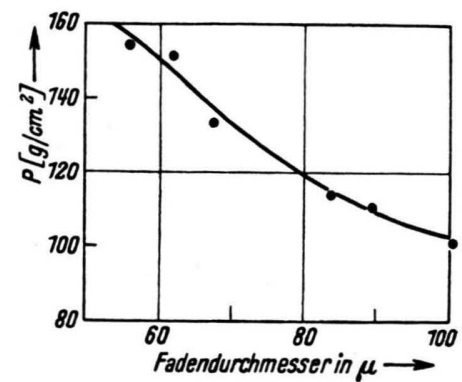

Abb. 2. Abhängigkeit der Maximalspannung von der Fadendicke. Mittelwert von 30 Fäden aus 8 Aktomyosinpräparaten (Bad wie in Abb. 1). T. $20^{\circ} \mathrm{C}$.

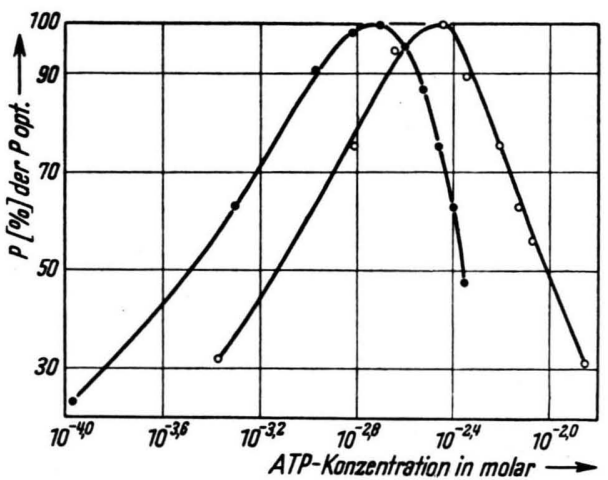

Abb. 3. Abhängigkeit der ATP-Kontraktion von der ATPKonzentration. - - - - F Fäden aus Aktomyosin von Anodonta. - - - - Fäden aus Aktomyosin vom Kaninchen (Bäder: $8 \cdot 10^{-4}-m . \mathrm{MgCl}_{2}, 8 \cdot 10^{-3}-m$. Phosphat, $\mathrm{KCl}$, Ionenstärke steigend mit der ATP-Konzentration von 0,07 bis $\left.0,16 \mu, p_{\mathrm{H}} \sim 6,9\right)$. T. $20^{\circ} \mathrm{C}$.

dete ATP-Konzentration nur halb so hoch wie beim Aktomyosinfaden aus Kaninchenmuskel. Eine höhere ATP-Konzentration kann nicht verwendet werden, weil die optimale ATP-Konzentration für das Fadenwie Fasermodell aus Anodonta nur halb so groß ist wie für die Modelle aus Skelettmuskel (Abb. 3) ${ }^{10}$.

9 G. u. M. U lbrecht, Z. Naturforschg. 7 b, 434 [1952].

10 A. We ber u. H. H. W e b e r, Biochim. biophysica Acta [Amsterdam] 7, 339 [1951].

11 W. H a s s elbach, Z. Naturforschg. 7 b, 163 [1952].

12 E. Heinz u. F. Holton, Z. Naturforschg. 7 b, 386 [1952].

\begin{tabular}{|c|c|c|c|}
\hline $\begin{array}{c}\text { Faden- } \\
\text { durch- } \\
\text { messer } \\
\text { in } \mu\end{array}$ & $\begin{array}{c}\text { Ver- } \\
\text { kürzungs- } \\
\text { zeit } \\
\text { in sec }\end{array}$ & $\begin{array}{c}\text { Verkürzungs- } \\
\text { geschwindigkeit in } \\
\text { or } \\
\text { der Anfangslänge } \\
\text { pro Sekunde }\end{array}$ & $\begin{array}{c}\text { Zahl der } \\
\text { Versuche }\end{array}$ \\
\hline & & 3,4 & 3 \\
125 & 14,8 & 2,1 & 5 \\
150 & 24,2 & 1,4 & 4 \\
175 & 35,8 & 1,1 & 1 \\
200 & 45 & 0,82 & 1 \\
225 & 61 & 0,67 & 1 \\
300 & 75 & & \\
\hline
\end{tabular}

Tab. 1. Abhängigkeit der maximalen Verkürzung von der Vorbehandlung der Fäden $\left(1,25 \cdot 10^{-3}-m\right.$. ATP, 0,04-m. $\mathrm{KCl}, 0,01-m$. Phosphat, $\left.8 \cdot 10^{-4}-m . \mathrm{MgCl}_{2}, p_{\mathrm{H}} \sim 6,9\right)$, T. $20^{\circ} \mathrm{C}$.

Die Lage des ATP-Optimums hängt also nicht nur von der Struktur *, sondern auch von der Art des betreffenden Aktomyosins ab.

Die maximale Verkürzung der unbelasteten Fadenmodelle beträgt knapp $70 \%$, wenn die Modelle in der üblichen Weise hergestellt wurden. Wird dagegen auf die Eiweißanreicherung durch partielle Verdunstung einer Glycerin-Wasser-Mischung verzichtet, so wächst der maximale Verkürzungsbetrag auf nahezu $80 \%$ (vgl. Tab. 1), d. h. auf den üblichen Verkürzungsbetrag aller Fasermodelle ${ }^{4}$. Der Vergleich zeigt, daß die üblichen Fadenmodelle sich nur infolge der partiellen Trocknung etwas weniger ausgiebig verkürzen als die Fasermodelle. Werden die Fäden nicht durch Dehnung vollständig orientiert, so ist das Verkürzungsmaximum wesentlich kleiner (Tab. 1).

\section{III.}

Die in Abb. 1 angegebenen Maximalspannungen gelten für $20^{\circ} \mathrm{C}$. Wie die Maximalspannung von der Temperatur abhängt, ist aus Abb. 4 zu ersehen. Diese Temperaturabhängigkeit ist anders als die Temperaturabhängigkeit des Fadenmodells aus Kaninchenmuskel ${ }^{7}$. Dagegen stimmen die Temperaturabhängigkeit des Faden- und des Fasermodells aus Anodonta vollständig überein.

* Daß auch die Struktur - und zwar die Packungsdichte - des Aktomyosins die ATP-Konzentration beeinflußt, oberhalb der die Wechselwirkung zwischen ATP und Aktomyosin wieder abnimmt, ist schon länger bekannt: So wird nach $\mathrm{H}$ a s s e l b a ch ${ }^{11}$ das Optimum der ATP-Spaltung durch Aktomyosinflocken (1-2\% Eiweiß) bereits mit einer ATP-Konzentration von $2 \cdot 10^{-3}-m$. unter den gleichen ionalen Bedingungen unterschritten, unter denen es bei der Spaltung durch Fibrillenmodelle erst bei $10^{-2}-m$. überschritten wird ( $\mathrm{H}$ e i n $\mathrm{z}$ und $\mathrm{Hol}$ to ${ }^{12)}$. 
Beide Anodonta-Modelle entwickeln bei $0^{\circ} \mathrm{C}$ nur etwa $40 \%$ der Spannung, die bei $15-20^{\circ}$ entwikkelt wird. Bei beiden Modellen fällt die Spannung, die bei $20^{\circ} \mathrm{C}$ erreicht ist, nur um etwa $20 \%$ wieder $\mathrm{ab}$, wenn das Präparat wieder auf $0^{\circ} \mathrm{C}$ gekühlt wird. Die Einstellung einer Gleichgewichtsspannung von oben und unten ist also für beide Arten von Anodonta-Modellen gleich unvollständig. Das ist der eine Unterschied gegenüber dem Faser- und Fadenmodell aus Kaninchenmuskel. Werden für beide Anodonta-Modelle die temperaturabhängigen Gleich-

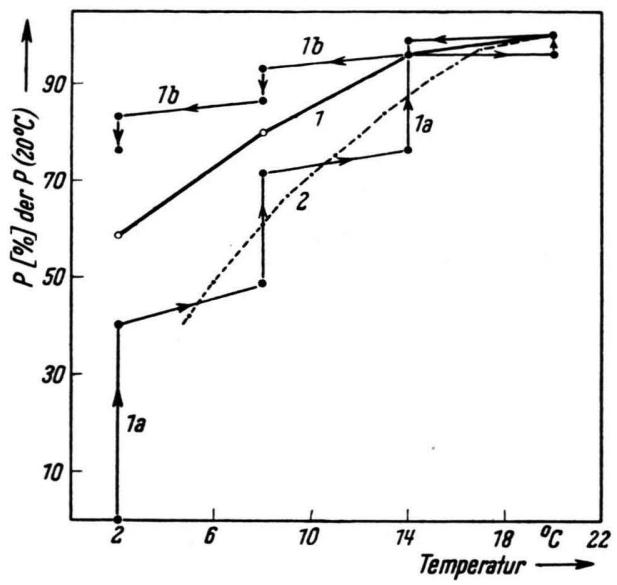

Abb. 4. Temperaturabhängigkeit der Spannung. Kurve 1, Anodontafaden: 1 a Einstellung bei steigender Temperatur, $1 \mathrm{~b}$ bei fallender Temperatur, 1 gemittelt aus $1 \mathrm{a}$ und $1 \mathrm{~b}$ (Bad wie in Abb. 1). Kurve 2, Temperaturabhängigkeit der Spannung des Fadens aus Kaninchenmuskeln $\left(2,5 \cdot 10^{-3}-m\right.$. ATP, 0,025-m. KCl, 0,007-m. $\mathrm{MgCl}_{2}$, 0,007-m. Phosphat, $\left.p_{\mathrm{H}} \sim 6,7\right)$.

gewichtsspannungen durch Interpolation ermittelt, so hängen sie für das Anodonta-Faden- und -Fasermodell in gleichem und außerdem wesentlich geringerem Umfang von der Temperatur ab als bei den Modellen aus Kaninchenmuskeln (Abb. 4).

Es sieht also so aus, als sei die verschiedenartige Abhängigkeit der Spannung von der Temperatur bei Kaninchenmuskeln einerseits und bei Anodonta-Muskeln andererseits nicht durch den komplizierten Bau der ganzen Muskelmaschine, sondern durch die Eigenschaften der beiden Aktomyosine bestimmt.

\section{IV.}

Wie alle lebenden Muskeln und alle Modelle zeigt auch der Aktomyosinfaden aus der glatten Anodonta-Muskulatur das bekannte „quick release“ $\mathrm{Phä-}$ nomen von $\mathrm{G}$ as ser und $\mathrm{Hill}{ }^{13}$.

\begin{tabular}{|c|c|c|c|}
\hline $\begin{array}{c}\Delta L \cdot 100 \\
L o\end{array}$ & $\begin{array}{c}L / L o \text { vor } \\
\text { dem release }\end{array}$ & $\begin{array}{c}\text { Halbwert- } \\
\text { zeit in sec }\end{array}$ & $\begin{array}{c}\text { Zahl der } \\
\text { Versuche }\end{array}$ \\
\hline $6,7 \%$ & $97 \%$ & 12 & 8 \\
$6,1 \%$ & $76 \%$ & 54 & 10 \\
$6,0 \%$ & $60 \%$ & 152 & 6 \\
\hline
\end{tabular}

Tab. 2. Halbwertzeit der Spannungsentwicklung nach "quick release“ bei verschiedener relativer Fadenlänge (Bad wie in Tab. 1$),$ T. $20^{\circ}$ C.

\begin{tabular}{|c|c|c|c|}
\hline \multicolumn{2}{|c|}{ Vorbehandlung der Fäden } & \multirow[b]{2}{*}{$\begin{array}{c}\text { Ver- } \\
\text { kürzung } \\
\text { um } \%\end{array}$} & \multirow[b]{2}{*}{$\begin{array}{c}\text { Zahl } \\
\text { der } \\
\text { Versuche }\end{array}$} \\
\hline $\begin{array}{c}\text { getrocknet, nach } \\
\text { Durchtränkung } \\
\text { mit } 30 \% \text { Glyzerin }\end{array}$ & $\begin{array}{c}\text { gedehnt um } \% \\
\text { der Anfangslänge }\end{array}$ & & \\
\hline - & $50 \rightarrow 100$ & 76 & 10 \\
\hline $12^{\mathrm{h}}$ & $50 \rightarrow 100$ & 66 & 6 \\
\hline $12^{\mathrm{h}}$ & - & 56 & 8 \\
\hline
\end{tabular}

Tab. 3. Abhängigkeit der Verkürzungsgeschwindigkeit von der Fadendicke. Mittlere Verkürzungsgeschwindigkeit für $50 \%$ Verkürzung $\left(1,5 \cdot 10^{-3}-m\right.$. ATP, $8 \cdot 10^{-4}-m . \mathrm{MgCl}_{2}$, $0,04-m$. KCl, 0,01-m. Phosphat $\left.p_{\mathrm{H}} \sim 6,9\right)$, T. $20^{\circ} \mathrm{C}$.

Die Spannungsentwicklung nach dem „release“ verläuft langsam (vgl. Tab. 2). Die Halbwertzeit der Spannungsentwicklung entspricht etwa der Halbwertzeit des Aktomyosinfadens aus Säugermuskulatur. Sie ist bedeutend größer als die Halbwertzeit der Spannungsentwicklung nach einem „release“ des langsamsten aller untersuchten Fasermodelle, d. h. des Fasermodells aus dem Kuhrectum ${ }^{9}$.

Der langsamen Spannungsentwicklung entspricht eine geringe Verkürzungsgeschwindigkeit. Die Verkürzung $100 \mu$ dicker Fäden um 50\% der Originallängen bei $20^{\circ} \mathrm{C}$ dauert $15 \mathrm{sec}$ anstatt $4,5 \mathrm{sec}$, in denen sich das Fasermodell aus Anodonta um 50\% verkürzt. Doch mögen die Verkürzungszeiten etwas zu hoch sein, weil die „Grenzschichtdicke“ für eine ATP-Konzentration von $1,5 \cdot 10^{-3}-m$. mit $100 \mu$ Dicke bereits überschritten ist. Dies geht unter anderem (vgl. II) auch daraus hervor, daß die Verkürzungszeiten mit steigender Dicke ebenfalls zunehmen (Tab. 3).

\section{V.}

Wird die ATP-Spaltung durch Vergiftung mit Salyrgan oder durch Entfernung des ATP aufgehoben oder aber durch Erhöhung der ATP-Konzentration auf überoptimale Werte stark eingeschränkt, so

13 H. Gasser u. A. V. Hill, Proc. Roy. Soc. [London], Ser. B 96, 398 [1924]. 
erschlafft auch der Aktomyosinfaden aus AnodontaMuskel, ebenso wie alle anderen Muskelmodelle, falls dafür gesorgt ist, daß der Faden nicht in Starre fällt (Abb. 5, 6, 7). Die Starre wird in den Versuchen, in denen die ATP-Spaltung durch Eigenhemmung

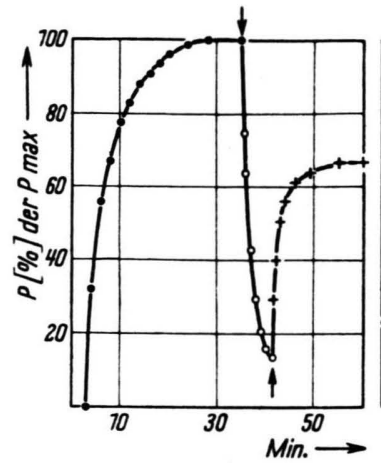

Abb. 5
Abb. 6

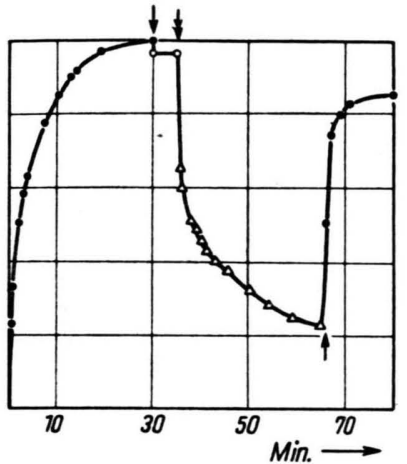

Abb. 5. Erschlaffung durch Salyrgan. -.-.- Kontraktion durch $1,25 \cdot 10^{-3}-m$. ATP, $\downarrow 2 \cdot 10^{-4}-m$. Salyrgan, $\uparrow 1 \cdot 10^{-2}$ $m$. Cystein (sonst wie in Abb. 1). T. $20^{\circ} \mathrm{C}$.

Abb. 6. Erschlaffung des kontrahierten ATP-freien Fadens

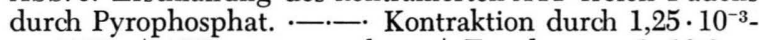
$m$. ATP, $\downarrow$ ATP ausgewaschen, $\$$ Zugabe von $1 \cdot 10^{-2}-m$. Pyrophosphat, $\uparrow$ Pyrophosphat ausgewaschen und 1,25 $\cdot 10^{-3}-m$. ATP zugegeben (sonst wie in Abb. 1). T. $20^{\circ} \mathrm{C}$.

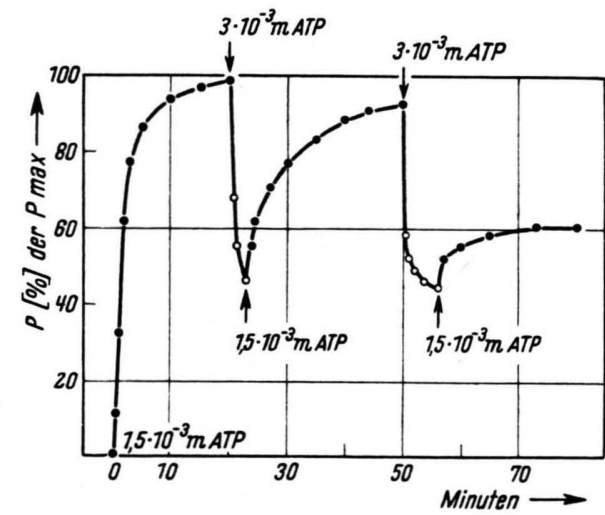

Abb.7. Die Abhängigkeit der Kontraktion und der Erschlaffung von der ATP-Konzentration (Ionalität wie in Abb. 1). T. $20^{\circ}$ C. -

des Substrates oder Gift „gehemmt" wird, durch die Weichmacherwirkung des anwesenden ATP vermieden (Abb. 5 und 7). Falls die ATP-Spaltung durch Auswaschen des ATP beendet wird, muß als Weichmacher ein anderes Polyorthophosphat, wie z. B. Pyrophosphat, hinzugefügt werden (Abb.6).

Die Erschlaffung des Fadenmodells aus AnodontaMuskel unterscheidet sich von der Erschlaffung aller Modelle aus Skelettmuskel dadurch, daß Pyrophos-
phat-Konzentrationen $>10^{-2}-m$. auch dann erschlaffend wirken, wenn ATP zugegen und die Mg-Konzentration $<10^{-3}-m$. ist (Abb. 8, 9). Hierin gleicht

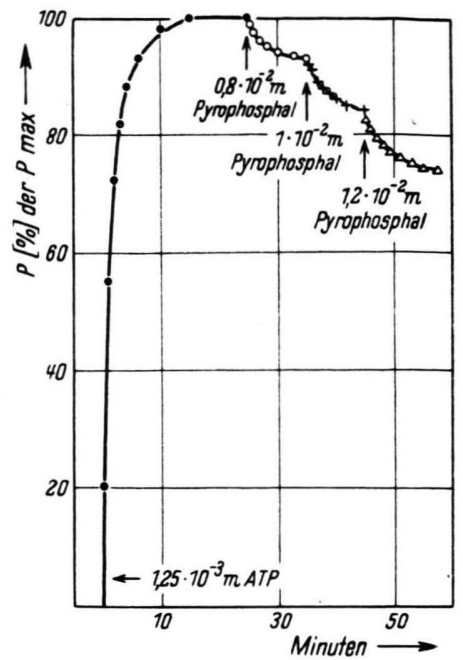

Abb. 8. Hemmung der ATP-Kontraktion durch Pyrophosphat (Ionalität wie in Abb. 1). T. $20^{\circ} \mathrm{C}$.

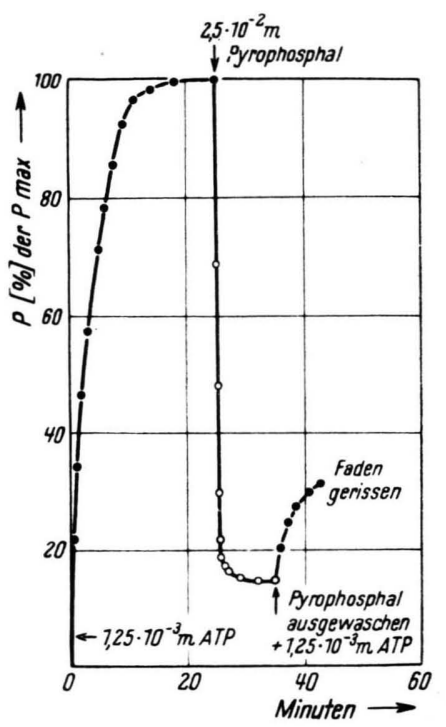

Abb. 9. Die irreversible Hemmung durch hohe Pyrophosphatkonzentration (Ionalität wie in Abb. 1). T. $20^{\circ} \mathrm{C}$.

das Fadenmodell dem Fasermodell aus Anodonta ${ }^{9}$. Beide Anodontamodelle gleichen sich auch darin, daß Pyrophosphatkonzentrationen $>2 \cdot 10^{-2}-m$. irreversible Schädigungen hervorrufen, die durch Auswaschen nicht $\mathrm{zu}$ beseitigen sind (Abb. 3 und vgl. Ulbrecht ${ }^{9}$ ). So scheint die eigenartige Pyrophosphat-Empfindlichkeit der Modelle aus glatten Muskeln nicht eine Eigenschaft ihrer Struktur, sondern ihres Aktomyosins zu sein. 
VI.

Die Ergebnisse zeigen, daß die Fadenmodelle aus gereinigtem Aktomyosin des glatten Anodonta-Muskels den Fadenmodellen aus Kaninchen-Aktomyosin in all den Eigenschaften gleichen, die für alle Muskelmodelle gleich sind: Verkürzungsmaximum, Giftempfindlichkeit der ATP-Kontraktion, Kontraktion bei Spaltung des ATP, Weichmacherwirkung und Erschlaffung bei Hemmung der Spaltung.

Die beiden Fadenmodelle gleichen sich darüber hinaus in ihrer geringen Zerreißfestigkeit, geringen maximalen Spannung und geringen Verkürzungsgeschwindigkeit. Diese Gleichartigkeit der Fadenmodelle kann entweder darauf beruhen, daß die Aktomyosinpartikel nicht mehr — hoch konzentriert - in die physiologische Faser- und Fibrillenstruktur eingebaut sind oder andererseits auf einer gewissen Schädigung der Aktomyosinpartikel selbst durch Extraktion und Umfällung.

Die beiden hier verglichenen Arten von Fadenmodellen sind untereinander verschieden in einer Peihe von Eigenschaften, in denen auch die Fasermodelle und die Muskeln selbst verschieden sind, aus denen das Aktomyosin der Fäden entnommen ist. Diese Eigenschaften sind die Temperaturabhängigkeit der Spannung, die Vollständigkeit oder aber Unvollständigkeit der Einstellung des temperaturabhängigen Gleichgewichts der Spannung, die Lage des ATP-Optimums, die Hemmbarkeit der ATPKontraktion durch Pyrophosphatkonzentration $>10^{-2}$ molar.

In allen diesen Eigenschaften gleicht jedes der beiden Fadenmodelle den Fasermodellen gleicher Herkunft - und zwar quantitativ. Das spricht dafür, daß diese Eigenschaften in den entsprechenden Fasermodellen und Muskeln verschieden sind, weil die Aktomyosine verschieden sind, und nicht, weil die übergeordneten Strukturen der Muskeln sich unterscheiden, in die diese Aktomyosine eingebaut sind.

14 H. Portzehl, G. S chramm u. H. H. Web e r, Z. Naturforschg. 5 b, 61 [1950].
Methodischer Teil

Die Herstellung der Aktomyosinlösungen

Ausgangsmaterial ist der Fußmuskel von Anodonta celensis. Er wird mit der zwei- bis dreifachen Menge dest. Wassers bei einer Temperatur $\sim 0^{\circ} 4 \mathrm{~min}$ im W a ring-Blender zerkleinert. Die Suspension wird dann mit $\mathrm{KCl}$ auf eine Ionenstärke von $0,6 \mu$ eingestellt und mit $1 / 100-m$. Phosphat prim./sek. $=1,5$ auf $p_{\mathrm{H}} \sim 7$ gepuffert und in Gegenwart von $5 \cdot 10^{-3}-m$. KCN 12 Stdn. bei $3^{\circ} \mathrm{C}$ extrahiert. Der Extrakt wird $30 \mathrm{~min}$ mit $22000 \mathrm{~g}$ bei $3^{\circ} \mathrm{C}$ klar zentrifugiert. Seine Eiweißkonzentration ist dann $\sim 1,2 \%$ und seine ATP-Empfindlichkeit

$\frac{\log \eta_{\text {rel }}}{\log \eta_{\text {rel ATP }}} \cdot 100{ }^{14}$ beträgt $\sim 100 \%$.

Die erste Fällung tritt dann, wenn kein ATP zugesetzt wird - anders als bei der Umfällung von KaninchenAktomyosin -, erst nach 15-20 min ein. Wird ATP zugesetzt, so wird die Fällung noch weiter verspätet. Der ATP-Gehalt des Extraktes ist auch nach der Verdünnung auf $0,06 \mu$ offenbar immer noch überoptimal. Der Niederschlag der 2. Umfällung wird mit $1 \times 10^{-4}-\mathrm{m}$. ATP superpräcipitiert und dadurch konzentriert.

Dieser zweite Niederschlag wird durch Zugabe von $10^{-2}-m$. ATP-Lösung $(1 / 4$ bis $1 / 5$ des Niederschlagvolumens) und von festem $\mathrm{KCl}$ gelöst und auf eine Ionenstärke von $0,6 \mu$ gebracht. Sie enthält dann $2-2,54 \%$ Eiweiß und wird nach der Spaltung des ATP gelartig.

\section{Die Herstellung der Aktomyosinfäden}

Sie erfolgt in üblicher Weise ${ }^{7}$ durch Einspritzen in ein Bad von $p_{\mathrm{H}} 6,9$ und einer Ionenstärke von $0,06 \mu$ mit einem Gehalt von $8 \cdot 10^{-4}-m . \mathrm{MgCl}_{2}$. Die Fäden können nach einem Aufenthalt von $20 \mathrm{~min}$ im Bad in $80 \%$ Glycerin $(d=1,23)$ übertragen werden, das mit $m / 100$-Phosphat auf $p_{\mathrm{H}} 6,8-7,0$ gepuffert ist.

Vor der Übertragung wurde der Eiweißgehalt der Fäden in der Regel - aber nicht immer (vgl. Tab. 1) erhöht, indem die Fäden mit 30-proz. wässeriger Glycerinlösung getränkt und das Wasser bei $4^{\circ} \mathrm{C}$ weitgehend verdunstet wurde. Der Eiweißgehalt beträgt dann $\sim 5 \%$.

Die glyceringetränkten Fäden werden unmittelbar vor dem Versuch - eingespannt in das Tensiometer - unter Kontrolle der Spannung und der Doppelbrechung bei $p_{\mathrm{H}} 6,9$ und einer Ionenstärke von $0,06 \mu$ annähernd auf das Doppelte gedehnt.

Die Technik der Versuche selbst ist die gleiche wie mit Fäden aus Kaninchenmuskel 7. 